\title{
COMMENTARY
}

\section{A Student's Perspective on Dismantling Racial Bias in Pharmacy School Education}

\author{
Sarah Temi Sofeso, BS, Sheila Mohebbi, BA, Laura Rambaran, BS, and Anahit Tatarian, BA \\ University of California, San Francisco School of Pharmacy, San Francisco, California
}

Corresponding Author: Sarah Temi Sofeso, University of California, San Francisco School of Pharmacy, 505 Parnassus Ave., San Francisco, CA 94143. Tel: 951-323-5156. Email: $\underline{\text { SarahTemi.Sofeso@ucsf.edu }}$

Submitted April 22, 2021; accepted July 23, 2021; ePublished August 2021

\begin{abstract}
It is well-established that race is a social construct that has little to no biological relevance in the absence of context such as the significant impact of the social, political, and environmental systems that contribute to health and health inequities. However, pharmacy school curricula often misrepresent race as the basis of disease diagnosis and reinforce race-based clinical guidelines without contextualization. Pharmacy schools, through the partnership of students and faculty, should 1) contextualize the mention of race and the differences in disease burden, and 2) provide evidence for race-based guidelines and clinical decision-making in education materials. In this way, we can work to halt the perpetuation of teaching bias to future healthcare professionals.
\end{abstract}

Keywords: pharmacy education, curriculum, race, health disparities, bias

\section{INTRODUCTION}

The completion of the Human Genome Project in 2003 demonstrated that human beings share 99.9\% of our DNA with each other regardless of race. ${ }^{1}$ Despite these overwhelming similarities, race-based diagnostic practices and treatment guidelines still exist in healthcare today. ${ }^{2}$ In "Misrepresentations of Race - The Role of Medical Schools in Propagating Physician Bias," Amutah and colleagues explore the ways in which medicine (often incorrectly) thinks about race and how those thinking patterns uphold inequities pervasive within medicine. ${ }^{3}$ The authors examined medical school curricula and highlighted misrepresentation of race in the following domains: semantics, prevalence without context, race-based diagnostic bias, the pathologization of race, and race-based clinical guidelines. Based on these areas, Amutah and colleagues provided recommendations on how to work towards undoing these deeply-rooted teachings. Though the authors center their discussion around medical school curricula in the United States, the issues raised are equally as important and can be directly applied to pharmacy education.

Didactic curricular content lays the foundation for the practices and decision-making skills students will use as they become pharmacists. Unfortunately, the way curricular content is taught often reinforces pre-existing beliefs and perceptions about race rather than challenging them. When practicing clinical vignettes in class, cases often note the patient's age, gender, and race followed by presenting symptoms and past medical history. This fleeting introduction of race, without context, facilitates the conflation of race and disease state, such as Black patients with heart failure and white patients with cystic fibrosis. While these associations are based on disease prevalence, they encourage ideas regarding innate biological differences between different races while failing to recognize diseases can affect individuals of any race. Students may potentially use race as a diagnostic shortcut, and if this is not addressed and corrected, it can perpetuate racism in healthcare as the student becomes the provider. It is instrumental that race is discussed in a nuanced manner that reflects the complexity and intersecting systems that contribute to a patient's health. The recent transition to an integrated, three-year, block-based curriculum and a call to action by the student body prompted the University of California, San Francisco (UCSF) School of Pharmacy (SOP) to analyze how race is represented within its curriculum with the hope of enhancing teaching through an antiracist framework. The UCSF SOP is not alone in this effort but given the sociopolitical climate and disproportionate impact of the COVID-19 pandemic on Black, Indigenous, and People of Color communities, we believe it is crucial for all Schools of Pharmacy to examine how the misuse of race in their curricula can propagate health inequities.

\section{Addressing Race in Pharmacy Education}

It is well-established that race is a social construct that has little to no biological relevance in the absence of context such as the significant impact of the social, political, and environmental systems that contribute to health and health inequities. ${ }^{4}$ Providing this context illustrates that differences in social experiences rooted in systemic and structural racism inflict biological consequences and not vice versa. Stating a patient's race without context perpetuates race as a 
risk factor for disease. As a student, it is both a jarring and frustrating experience to attend lectures or read cases where racial/ethnic differences in the incidence of disease are attributed to genetic predispositions, particularly when we identify with the race or ethnicity mentioned. One example would be if a lecture stated, without context, that human immunodeficiency virus (HIV) disproportionately affects Black and Latina women in the United States. ${ }^{5}$ If this was the only information presented, learners may assume there is a genetic predisposition for acquiring HIV. While it is important to acknowledge health disparities exist, it is crucial to scaffold learning by discussing the root causes of disparities such as mistreatment by healthcare systems, lower access to HIV preventative resources and care, and socioeconomic barriers.

For instance, Black women are linked to care at lower percentages than their white counterparts and have lower rates of pre-exposure prophylaxis use primarily due to a lack of targeted messaging and thus unawareness in their communities. ${ }^{6}$ It is important students understand these correlations between race, access to care, and prevalence of HIV. We encourage educators to be intentional and explicit with their use of race/ethnicity across all curricular materials by using geographic ancestral origin when discussing genetic risk and ensuring consistent contextualization. In clinical cases, if the educational goal is not aimed at addressing racism as a social determinant or equipping learners with the knowledge and resources on how to work towards addressing gaps in healthcare, race need not be mentioned. However, it is crucial that pharmacy educators seize every opportunity to discuss the social and structural factors that contribute to health inequities and create space where both students and faculty alike can discuss how our biases, when unchecked, create harm for our patients.

As pharmacy students, we feel particularly compelled to address structural racism embedded in clinical guidelines used to inform therapeutic decision-making. Amutah and colleagues recognize that "research conducted with a flawed understanding of race informs flawed guidelines." ${ }^{\text {"3 }}$ Race-based guidelines can incorrectly reinforce inherent biases of students and practitioners and negatively impact the health of patients. A notable example of this is the 2017 American College of Cardiology/American Heart Association (ACC/AHA) hypertension guidelines that discourage use of ACE inhibitors and ARBs in Black patients despite their efficacy in all patients as first-line hypertensive agents. ${ }^{7}$ A clinical case presented to students highlights the discrepancy in treatment decisions between Black and non-Black patients based on these guidelines:

"Because MK is black and does not have CKD, thiazide-like diuretics or CCBs are first-choice antihypertensive therapy. If $M K$ is not Black, ACEI and ARB would also be optimal alternatives."

This type of language discourages learners from evaluating patients holistically and diminishes the meaning of patient-centered care. If a race-based recommendation is mentioned, not only should evidence for the statement be inextricably provided, but opportunities must also be presented to challenge such statements, either through a critical appraisal of the scientific evidence that resulted in those guidelines, established counterevidence, or a discussion or debate of the recommendation. For instance, the clinical case above could be enhanced as follows:

"The 2017 ACC/AHA hypertension guidelines state that thiazide-type diuretics and CCBs are superior to ACE inhibitors and ARBs in Black patients and are thus recommended as first-line hypertensive agents in this population. However, while the 2002 ALLHAT trial upon which these recommendations are based declares a statistically significant lower incidence in the secondary outcomes of combined cardiovascular disease, stroke, and heart failure with chlorthalidone versus lisinopril in Black patients, these statements were discordant with subgroup analysis data showing that the benefit in combined cardiovascular disease and heart failure were the same in the Black and non-Black subgroups. Additionally, while there did appear to be a benefit in stroke reduction with chlorthalidone in Black patients, this benefit was seen in all trial participants, regardless of race. Furthermore, the ACC/AHA guidelines fail to mention that there was no difference between any agent in the prevention of the primary outcome (prevention of combined fatal coronary heart disease or nonfatal myocardial infarction) in all trial participants. Thus, a critical evaluation of the scientific literature indicates that MK would benefit from any first-line hypertensive agent despite what is plainly stated in the 2017 ACC/AHA guidelines."

We believe it is the duty of pharmacy educators to help guide students' critical evaluation of race-based guidelines for the betterment of our patients' health. We ask that pharmacy educators deeply examine how they present and teach therapeutic concepts involving race and adopt the transformative framework of critical pedagogy that advocates for understanding the intersectional systems that affect an individual's health and actively working to dismantle them. ${ }^{9}$ Without a bold change in the thought processes and conversations we have around therapeutics and race, future healthcare providers run the risk of making poorly-informed treatment decisions based solely on race to the detriment of our patients. 
As learners with rich and diverse backgrounds and experiences, we view the pharmacy curriculum from a variety of unique perspectives. We interpret clinical cases in ways that might not have been intended by case authors. This understanding led to the formation of a student-run Health Equity Curriculum Intern group, supported by the UCSF SOP, with faculty oversight. As Health Equity Curriculum Interns, we believe our role in supporting the utilization of an antiracist and health equity framework is vital. Interns work with faculty and course directors to analyze patient cases presented in the curriculum to ensure each patient's race, gender, and other protected characteristics are appropriately contextualized to avoid contributing to harmful stereotypes. In doing so, we can highlight missed opportunities to discuss social determinants of health, their structural causes, and how they contribute to disparities among minority groups.

Through a direct line of communication, we can see our suggestions to course directors be considered and implemented in real-time for the benefit of future students. Additionally, we have worked with faculty to facilitate health equity discussions amongst pharmacy students to promote recognition of our inherent biases and awareness of the marginalization and privileges within healthcare. Although our work as interns has been well-received and impactful, students cannot continue to be the only catalysts for change. It is imperative that pharmacy educators and leadership recognize their responsibility in advocating for these changes as well.

\section{Conclusion: A Call to Action}

We understand that there are a multitude of barriers that those in positions of leadership may feel when thinking about implementing the changes needed to achieve an antiracist and health-equity-focused curriculum. We recognize that faculty may be uncertain of where to begin making these changes or feel ill-equipped due to a lack of expertise in topics surrounding diversity, equity, and inclusion. We also acknowledge the fear of failure when trying to implement new practices and policies without having the resources and support to do so. Nonetheless, it is within the oath of a pharmacist to protect the welfare of humanity. We have a responsibility to work towards dismantling systems of oppression that uphold health inequities. No one is an expert in this area, which is why we must approach this work with the framework of cultural and structural humility. Although this will require extensive time and commitment to implement new programs or review materials in a set curriculum, these changes are necessary to start reform and challenge our biases in order to be better pharmacists, clinicians, students, teachers, and members of society.

We primarily base our editorial on two recommendations made by Amutah and colleagues, but this is only a starting point. Other recommendations by Amutah and colleagues include the standardization of language used to describe race/ethnicity and changing the way race is used to generate and access medical knowledge. Additionally, we emphasize the dismantling of racial bias in this article, but we recognize that other biases exist in terms of gender identity and sexual orientation that transverse the pharmacy field and should be challenged in similar ways. We implore pharmacy educators to begin taking steps to contextualize the mention of race and the differences in disease burden, and to provide evidence for race-based guidelines and clinical decision-making. Pharmacy educators are encouraged to search for other articles on this topic that resonate with their teaching philosophy and to explore resources such as MedEdPortal to inspire the creation of antiracist pharmacy educational activities. We emphasize the value of student voices, insight, and perspectives when making these changes. As students, we experience the curriculum and the changing world in a way our faculty might not. Collectively, with students and faculty working together, progress can be made towards more equitable healthcare.

\section{Acknowledgements}

The authors gratefully acknowledge Jennifer Cocohoba, PharmD, MAS, BCPS, AAHIVP and Stephanie Hsia, PharmD, BCPS, BCPP for their assistance in the writing and technical editing of this manuscript. The authors report no financial disclosures or conflicts.

\section{References}

1. Foster MW, Sharp RR. Beyond race: towards a whole-genome perspective on human populations and genetic variation. Nat Rev Genet. 2004 Oct;5(10):790-6. doi: 10.1038/nrg1452. PMID: 15510170.

2. Ioannidis JPA, Powe NR, Yancy C. Recalibrating the Use of Race in Medical Research. JAMA. 2021;325(7):623624. doi:10.1001/jama.2021.0003

3. Amutah C, Greenidge K, Mante A, et al. Misrepresenting Race - The Role of Medical Schools in Propagating Physician Bias. N Engl J Med. 2021;384(9):872-878. doi:10.1056/NEJMms2025768 
4. Gee GC, Ford CL. STRUCTURAL RACISM AND HEALTH INEQUITIES: Old Issues, New Directions. $D u$ Bois Rev. 2011;8(1):115-132. doi:10.1017/S1742058X11000130

5. "Black Americans and HIV/AIDS: The Basics." Kaiser Family Foundation, 7 Feb. 2020, www.kff.org/hivaids/fact-sheet/black-americans-and-hivaids-the-basics/.

6. Bradley ELP, Geter A, Lima AC, Sutton MY, Hubbard McCree D. Effectively Addressing Human Immunodeficiency Virus Disparities Affecting US Black Women. Health Equity. 2018;2(1):329-333. Published 2018 Nov 16. doi:10.1089/heq.2018.0038

7. Whelton PK, Carey RM, Aronow WS, et al. 2017 ACC/AHA/AAPA/ABC/ACPM/AGS/APhA/ASH/ASPC/NMA/PCNA Guideline for the Prevention, Detection, Evaluation, and Management of High Blood Pressure in Adults: A Report of the American College of Cardiology/American Heart Association Task Force on Clinical Practice Guidelines [published correction appears in Hypertension. 2018 Jun;71(6):e140-e144]. Hypertension. 2018;71(6):e13-e115. doi:10.1161/HYP.0000000000000065

8. ALLHAT Officers and Coordinators for the ALLHAT Collaborative Research Group. The Antihypertensive and Lipid-Lowering Treatment to Prevent Heart Attack Trial. Major outcomes in high-risk hypertensive patients randomized to angiotensin-converting enzyme inhibitor or calcium channel blocker vs diuretic: The Antihypertensive and Lipid-Lowering Treatment to Prevent Heart Attack Trial (ALLHAT) [published correction appears in JAMA 2003 Jan 8;289(2):178] [published correction appears in JAMA. 2004 May 12;291(18):2196]. JAMA. 2002;288(23):2981-2997. doi:10.1001/jama.288.23.2981

9. Cavanagh A, Vanstone M, Ritz S. Problems of problem-based learning: Towards transformative critical pedagogy in medical education. Perspect Med Educ. 2019;8(1):38-42. doi:10.1007/s40037-018-0489-7 